\title{
Innate immune cell responses in non pathogenic versus pathogenic SIV infections
}

Nicolas Huot ${ }^{1,2,3^{*}}$, Philippe Rascle ${ }^{1,3^{*}}$, Thalia Garcia Tellez ${ }^{1}$, Beatrice Jacquelin $^{1}$ and Michaela Müller-Trutwin ${ }^{1,3}$.

* co-first authors

${ }^{1}$ Unité HIV, Inflammation and Persistence, Institut Pasteur, Paris, France; ${ }^{2}$ CEA, Division of Immuno-Virology, iMETI, DSV, Fontenay-aux-Roses, France $;^{3}$ Vaccine Research Institute, Créteil, France.

Corresponding author: Michaela MULLER-TRUTWIN michaela.muller-trutwin@ pasteur.fr

Key words : natural hosts, NK cells, dendritic cells, innate lymphoid cells, neutrophiles 


\section{Introduction}

The infection of a host by a virus leads to one of the following three distinct outcomes: clearance of the virus, establishment of a chronic infection and the worst possibility, the death of the host. The quality and the duration of the host response, which may be influenced intrinsically (i.e. genetic background) or extrinsically (i.e. medication) engages the resolution of the infection toward one of those possibilities.

The host response can be divided intotwo main arms: the innate immune system and its orchestration of cells belonging to the adaptive immune system and the adaptive immune system itself. These different arms are interdependent and their actions are spatially and temporally organized in order to give an optimal response against the pathogen.

HIV infection is disturbing both the innate and adaptive arms of the host response. The introduction of the combined anti-retroviral treatment (cART) allows immune restoration, however to variable degrees. The capacity of the treatment to restore the functionality of the immune systemvaries depending on several factors, in particular the stage of infection at which the treatment is initiated.

Little is known on the impact of cART on the innate immune system. This lack of knowledge is partly due to the fact that patients were rarely detected for HIV during the acute phase of infection, at the moment where the inflammation and the innate response is the most active.In this context, the study of SIV infection in non human primates (NHP) remains a major model of choice to study the first steps of infection. Indeed, Asian species of NHP, such as rhesus and cynomolgus macaques, experimentally infected by SIV, experience a spectrum of disorders identical to HIV-1 infected humans. NHP models contributed to increase significantly our knowledge on HIV infection, for instance helped to apprehend the early establishment of the viral reservoirs and the immense immunological insult to the gut, as well as other tissues, given by the viral infection ${ }^{1-4}$.NHP from Africa, such as African green 
monkeys (AGM), sooty mangabeys (SM)and mandrills, are natural hosts of SIV. SIV infection in these natural hosts generally does not lead to any signs of disease, even though they carry high plasma and intestinal viral load ${ }^{5}$. One major difference between AIDS progressors and non-progressors is the duration of the inflammation, which is totally resolved in natural hosts by the end of the acute infection, while chronicinflammation persists in AIDS progressors (Figure 1).Inflammation is initially induced by cells that sense the virus. Subsequently, cells of the adaptive immune system, as well as other mechanisms, might contribute to chronic inflammation, for instance microbial translocation which is absent in natural hosts ${ }^{6-8}$.Indeed, natural hosts maintain the integrity of the intestinal barrier and do not loose their Th17 cells ${ }^{5,8,9}$. Another remarkable difference in the natural host is the preservation of the architecture of the secondary lymphoid tissues, the latter being progressively disrupted in HIV and SIVmac infections ${ }^{10,11}$. The preservation of such a structure could participate to the better orchestration of immune responses in natural hosts. This review will discuss in particular the hallmarks of innate immune responsesin natural hosts. Innate immunity is generally less well explored than $\mathrm{T}$ and $\mathrm{B}$ cell responses, except for dendritic cells and NK cells. We will here summarize the information that is available on innate immune responses in natural hosts and explain the major differences with respect to pathogenic HIV and SIV mac infections.

\section{Monocyte/Macrophages}

Monocytes and macrophages possess receptors for entry of HIV/SIV viruses, like dendritic cells and CD4 T cells. While primary monocytes are not susceptible to HIV infection, HIV infection of macrophages in tissues, such as the brain, lung and decidua, has been reported in

many studies ${ }^{12,13}$. It has been suggested that this cell population figures among the first target cells to be infected by SIV / HIV in vaginal mucosa ${ }^{13,14}$.PCR and 
immunohistochemistryanalyses of the central nervous system and lungs of AGM showed that macrophages are also infected in natural hosts ${ }^{15}$.Surprising results were however obtained during in vivo depletion studies. Upon depletion of CD4+ T cells in chronically infected SM, viremia declined $^{16,17}$. The latter finding contrasts with macaques in which upon CD4+ T cell depletion viremia increased, followed by quick progression to $\operatorname{AIDS}^{18,19}$. This increase in viral replication in CD4-depleted macaques was due to massive infection of macrophages, suggesting that natural host's macrophages are less susceptible to infection. The potentially lower susceptibility of macrophages to SIV infection has been further shown in monocytederived macrophages (MDM) in vitroand attributed to a higher expression of IFN- $\alpha$ inducible restriction factors, such as Tetherin and TRIM $22^{20}$. In line with this, immunohistochemistry of the intestine did not reveal any SIV-infected macrophages in AGM at the stage of peak viral production in early infection ${ }^{7}$.More studies need to be done at distinct stages of infection and in othertissues to better understand the SIV infection pattern of macrophages in natural hosts.

In macaques, AIDS and death can occur in the absence of detectable macrophage infection $^{21,22}$. These results could indicate that it is not the infection of monocyte/macrophages that matters but maybe more their role in inflammation. Soluble markers of inflammation and coagulation but not T-cell activation predict non-AIDS-defining morbid events during treatment ${ }^{23,24}$. Markers of monocyte activation (sCD14, TNF- $\alpha$, CXCL10/IP-10) and microbial translocation have been shown to be associated with disease progression in HIV infection and a poor $\mathrm{CD} 4+\mathrm{T}$ cell recovery under $\mathrm{ART}^{25,26}$. In treated patients, monocyte activation predicts coronary artery calcium progression ${ }^{27}$. Massive turnover of peripheral monocytes associated with death of tissue macrophages correlates with AIDS progression ${ }^{28}$ in the macaque model. Importantly, the level of monocyte turnover was a better predictive marker for AIDS progression than was viral load or lymphocyte activation ${ }^{29}$. 
In $\mathrm{SM}$, a reduced monocyte $\mathrm{TNF}-\alpha$ response was noted as compared to monocytes from humans and macaque ${ }^{30}$. Moreover, since microbial translocation is not occurring in natural hosts, their monocytes/macrophages are not exposed to PPS $^{31-33}$. In line with this, the monocyte/macrophage activation marker sCD14 is not increased in wild SIV-infected AGMs in contrast to macaques ${ }^{32}$. Altogether,these findings suggest that monocyte/macrophages are less activated in natural hosts than during HIV/SIVmac infections in human and macaques.

\section{Plasmacytoid dendritic cells}

Plasmacytoid dendritic cells (pDC) are rapidly mobilized by day 2 post-infection (p.i.), both in pathogenic and non pathogenic infection (Figure 1) ${ }^{34-36}$. Only in pathogenic infection, there is however a progressive decline of $\mathrm{pDC}$ numbers in the blood in chronic infection.

Data on the infection rates of pDCin vivo are scarce. One study reported the presence of HIV DNA in circulating pDC of chronically HIV-infected patients ${ }^{37}$. Another study reported high infection levels in lymph node (LN) pDC during acute SIVmac infection ${ }^{36}$. We have reported that AGM pDC express extremely low levels of CD4, unlike MAC and human $\mathrm{pDC}^{38}$. Moreover, both AGM and SM pDC were found to be, in contrast to MAC pDC, predominantly negative for CCR5. Despite such limited CD4 and CCR5 expression, lymphoid tissue $\mathrm{pDC}$ were infected to a similar degree as $\mathrm{CD}^{+} \mathrm{T}$ cells, both in MAC and $\mathrm{AGM}^{38}$

pDC form a rare cell population that ishowever responsible for the vast majority of IFN-I production after HIV/SIVmac encounter.To analyze whether pDC of natural hosts are also the predominant producers of IFN-I in response to SIV, PBMC from healthy AGM were depleted for $\mathrm{pDC}$ and stimulated with SIVagm. This led to a $96.7 \%$ reduction in IFN-I production upon stimulation with SIVagm, confirming the predominant role of pDC for SIV sensing in natural hosts as well ${ }^{34}$. pDCfrom natural hosts are fully functional to sense SIV ${ }^{34,39,40}$. In line with 
this, no significant differences are observed between pathogenic and non pathogenic SIV infections regarding the capacity to produce IFN-I in vivo ${ }^{39-42}$. The maturation and homing profiles of $\mathrm{pDC}$ in response to SIV infection in vivo were also similar between AGM and macaques ${ }^{41,42}$.pDC from AGM even show a higher capacity to sense species-pecific viruses (SIVagm) than viruses from experimental hosts (SIVmac), evoking host-virus adaptation and selective pressures to maintain the capacity of SIV sensing in the natural host ${ }^{38}$.

IFN-I producedduring acute infection strongly upregulates type I IFN-stimulated genes (ISGs) in both pathogenic and non-pathogenic SIV infection ${ }^{39,43,44}$. ISG expression returns, however,to basal levels after acute infection in natural hosts, while it remains sustained in macaques, as in HIV infection in humans. Since the capacity of IFN-I production is similar between natural hosts and macaques, the sustained ISG response during chronic SIVmac/HIV infections is most likely due to other factors than IFN-I, such as translocated microbial products or other factors ${ }^{39,42,45}$.

\section{Myeloid dendritic cells}

The kinetics of myeloid dendritic cell $(\mathrm{mDC})$ numbers are similar between non-pathogenic and pathogenic infection during the first year postSIVinfection (Figure 1) ${ }^{42}$. The numbers are sometimes significantly increased in the early stages of infection in both types of infection. Only in pathogenic infection though, declines of $\mathrm{mDC}$ can be observed afterwards.

The infection of $\mathrm{mDC}$ by SIV in natural hosts has not been explored. Vpx has been shown to increase the susceptibility of HIV-1 to replicate in myeloid cells ${ }^{46}$.However, while Vpx is present in viruses of some natural hosts (SIVsm), it is absent in others (SIVagm), and its impact in non pathogenic infection is unclear.

HIV-1 is known to activatepDC,which activation is necessary toinduce the bystander maturation of $\mathrm{mDC}$, that do not mature if exposed to HIV in the absence of $\mathrm{pDC}^{47}$. In vivo, 
mDC show a lower maturation profile in natural hosts than in HIV/SIVmac infections ${ }^{33,42}$. After infection, spontaneous production of proinflammatory cytokines by mucosal mDCs increased only in progressor macaques but not in natural hosts. These findings are consistent with a model in which myeloid cells are less activated in natural hosts than during pathogenic infection,although more studies are needed to confirm this hypothesis.

\section{Innate lymphoid cells}

Recently discovered innate lymphoid cells (ILC) are composed of three classes with their own specificities ${ }^{48-49}$. These cells are mainly located near the mucosal epithelial barriers. The population of ILC3 (NKp44+ and ROR $\gamma \mathrm{T}+$ ) producing IL-17 is lost during the acute phase of SIV infection in macaques ${ }^{3,450}$. IL-17 is essential for the recruitment of neutrophils at the inflammation site ${ }^{51}$. This fact might explain in part the neutropenia observed during acute phase of pathogenic HIV/SIV infections. In addition, it might be associated with thefragility of the intestinal barrier. The absence of bacterialtranslocation in the natural hostsraises the question about the role of ILCs in this model. ILC have not been characterized yet in the natural hosts.

\section{Polymorphonuclear cells}

Neutrophils are commonly known as "professional" phagocytic cells of the innate immune system $^{51,52}$. They are present in large numbers in the peripheral blood (50-70\% of white cells) and are recruited rapidly to peripheral sites of damage, where they can capture and kill microbes efficiently through the production of ROS (such as $\mathrm{O}_{2}{ }^{-}, \mathrm{H}_{2} \mathrm{O}_{2}, \mathrm{HOCl}$, and $\left.\mathrm{OH} \cdot\right)^{53}$. The role played by neutrophils in HIV/SIV infection has rarely been investigated ${ }^{53,54}$. An increase of PMN apoptosis has been shown in humans, particularly in the later stages of HIV disease ${ }^{53,55}$,but the mechanisms have not been identified. In NHP, PMN are identified as 
$\mathrm{CD} 45+\mathrm{CD} 11 \mathrm{~b}^{\text {high }} \mathrm{CD} 14^{\mathrm{lo56,57}}$.Two studies have shown a strong depletion of neutrophils, attributed to an increase of neutrophil apoptosis, during the acute phase of infection in macaques, whereas this depletion was not observed in the naturalhost (Figure 1$)^{56,57}$.

Besides producing cytokines and chemokines, another novel and emerging aspect consists in the ability of neutrophils to interact with, and modulate the activity of different leukocyte types in vitro and in vivo. Recent studiesalso highlight a new role for neutrophils as nonredundant regulatory cells ensuring the terminal maturation of NK cells in both humans and mice $^{58-60}$. Future studies in natural hosts will help to decipher to which extent these functions play a role in the pathogenic outcome of HIV/SIV infections.

\section{Invariant Natural killer T cells (iNKT)}

A small subset of T lymphocytes named NKT cells express surface markers characteristic of both $\mathrm{T}$ cells and NK cells. They are known to play an important link between innate and adaptive immune responses ${ }^{61,62}$. Despite the low frequency of the iNKT population in the periphery $(<1 \%$ of the $\mathrm{CD} 3+$ cells $)$, the role $\mathrm{iNKT}$ in various pathologies such as allergy, cancer and infectious diseases is well considered.

In humans and NHP models, iNKT cells are characterized by the expression of a TCR comprised of V $\alpha 24-\mathrm{J} \alpha 18$ associated with $\mathrm{V} \beta 11^{63}$. The majority of iNKT express CD161 and all respond to lipid ligands through CD1d restriction. In Humans and macaques, iNKT express CD4 and CD8 $\alpha$, allowing the iNKT subset to be defined as $\mathrm{CD}^{+}$, DN (CD4-CD8-), or $\mathrm{CD}^{+64-67}$. In SM,iNKT cells are either $\mathrm{CD} 8^{+}$or DN, and never $\mathrm{CD} 4^{+68,69}$.Studies of iNKT in NHP highlight the importance of iNKT activation during HIV-1 infection. Infection of macaques resulted in peripheral CD4+ iNKT depletion similar to human HIV-1 infection, and was correlated with CD4+T cell decline. Moreover iNKT numbers were inversely correlated with viral load ${ }^{67,68}$. 
The macaque model also revealed an early expansion of IL-17 expressing CD4 ${ }^{+} \mathrm{NKT}$ cells in peripheral and mesenteric lymph nodes ${ }^{70}$.This could be a compensatory mechanism for the loss of other IL-17 producing cells such as Th17 cells.In contrast, in the nonpathogenic model, no change in the level of IL-17-expressing cells was observed in these tissues by immunohistochemistry. Consistent with the emergence of TGF- $\beta$ and IL-18 during the acute phase in SIV-infected macaques, but not in SIV-infected AGM, in vitro TGF- $\beta$ and IL-18 induced the differentiation and expansion of $\mathrm{IL}-17^{+} \mathrm{NKT}^{70}$.

SM iNKT have the capacity to produce IL-2,IL-13, and IL-10, which couldprovide these cells a role in the regulation of inflammation ${ }^{67}$. Indeed in murine models, the production of IL-4 and IL-10 by iNKT can induce regulatory T cell (Treg) development ${ }^{71}$. Alternately, the data decribed in SM also raise the possibility that loss of anti-inflammatory NKT function promotes chronic immune activation in pathogenic SIV infection, while intact NKT function helps to protect natural hosts from developing immunodeficiency and aberrant immune $\operatorname{activation}^{68}$.

\section{Natural killer cells}

NK cells were thought to be a primitive cell lineage, which evolved prior to the development of the adaptive immune system. Recently, however, NK cells have been shown to also possess traits of adaptive immunity and to acquire immunological memory in primates ${ }^{72}$.

Healthy human subjects typically exhibit three distinct NK cell subsets that are defined by the differential expression of CD56 and CD16 within the $\mathrm{CD}^{-}$population. Following HIV infection, a significant decline occurs in the cytolytic subset $\left(\mathrm{CD} 56{ }^{\mathrm{dim}} \mathrm{CD} 16+\right)$ while an expansion of the otherwise rare population of $\mathrm{CD} 56^{-} \mathrm{CD} 16^{+} \mathrm{NK}$ cells occurs. Alterations in the levels of $\mathrm{CD}^{-} \mathrm{CD}^{- \text {bright }} \mathrm{CD}^{-}$cells do not appear to be major but reduced cytokine and chemokine production has been reported. These and other data demonstrated that in addition 
to adversely affecting the adaptive immune response, HIV-infection also results in functional impairment of the NK cell compartment of the innate immune system.

Studies in SIV infected rhesus macaques also highlight multiple effects ofinfection on NKcell function, suchas a decreased ability to secrete IFN- $\gamma$, TNFaand IL-2. NK cells in SIV infected rhesus macaqueswith rapid disease progression and high viral loadshow sustained proliferation but signs of exhaustion and functional anergy ${ }^{73}$.

In natural hosts, the NK cell responses appear earlier and stronger than in SIVmac-infected macaques during the acute phase ${ }^{42,74}$. The cytolytic subset is not depleted and sometimes even seems to expand. The rapid and strong increase in NK cell proliferation might be a direct consequence of the early and robust production of IL-15 and IFN- $\alpha$ during primary SIVagm infection $^{42}$. It has been shown that IFN- $\alpha$ and IL-15 promote NK cell proliferation and survival, while IFN- $\alpha$ is able to increase NK cell cytotoxicity, and IL-15 to augment the secretion of IFN- $\gamma^{76}$.In natural hosts, despite the induction of both cytokines (IFN-a, IL-15) during the acute phase of SIV infection, no ex vivoproduction of IFN- $\gamma$ by NK cells could be observed $^{42}$. Interestingly though, the expression of CD107a on NK cells was increased in lymph nodes during the acute phase of SIVagm infection. An increase of CD16+CD56+ NK cells, generally considered as the cytolytic subset of NK cells, has also been described in blood of SIV-infected $\mathrm{SM}^{74}$. These few studies on NK cells in natural hosts suggest that they display a stronger cytotoxic activity in response to SIV infection than during SIVmac infection.

\section{Conclusions}

Many eventsoccur simultaneouslyafter HIV infection with very tightly regulatedinteractionsbetween the distinct components ofthe immune system. A better understanding of the cross-talks between these cells and their impact on control of viral 
replication and disease is needed. For instance, more knowledge is desirable on the contribution of myeloid cells on inflammation during HIV/SIV infections. It is also unclear, to which extent specific cell types of the innate immune system, in particular macrophages or myeloid dendritic cells, are targeted by the virus in vivo.

Moreover, several other cells have been understudied so far. For instance, ILCs might play an important role during HIV/SIV infections, particularly in the intestine.An IL-17-mediated crosstalk between ILCs and neutrophils shows another type of possiblecommunicationbetween cells.Other less explored cells, such as iNKT,have the capacity to induce many anti-inflammatory cytokines and have a potential role in regulating locally the immune system. Additional innate cells exist that have been poorly or not at all investigated in HIV/SIV infections ${ }^{77,78}$.

NK cells have been largely studied in humans and macaques, but only poorly in natural hosts. NK are the only immune cells, thatwere stronger activated in natural hosts than in pathogenic infection $^{42,74}$. Thus, NK cells might play a so far unknown role in the lack of disease progression in natural hosts.

The recent description of the full genome of African green monkeys, as well as future whole genome sequence information from other natural hosts will help to develop more tools to analyse innate immune cells $\mathrm{s}^{79,80}$. Finally, studies on the metabolic changes due to the stress induced by the viralinfection within innate immune cells might reveal new aspects of immune and viral regulation ${ }^{81,82}$.

Studies in natural hosts have contributed to pinpoint that early control of infection is crucial. For instance in natural hosts for SIV, the inflammation is rapidly resolved, by the end of acute infection. Somepatients showed that receiving a treatment in early HIV infection canlead to control the virus after treatment interruption ${ }^{83}$. A limitation of virus replication in early time points of infection enhances the capacity of the hosttocontrolthe infection. It will be important 
to understand to what extent an early anti-retroviral treatment impacts the function of the innate immune system and the intercellular communications. Further studies in natural hosts should help to distinguish those early events which are associated or not with a good outcome of infection ${ }^{1}$.

\section{Acknowledgments}

We would like to thank the French National Agency for Research on AIDS and Viral Hepatitis (ANRS), the «Investissements d'Avenir program » managed by the ANR under reference ANR-10-LABX-77, as well as the Center for Infectious Disease models and Innovative Therapies (IDMIT) for their support.

\section{References}

1. Micci, L. et al. Interleukin-21 combined with ART reduces inflammation and viral reservoir in SIV-infected macaques. J. Clin. Invest.125, 4497-4513 (2015).

2. Whitney, J. B. et al. Rapid seeding of the viral reservoir prior to SIV viraemia in rhesus monkeys. Nature512, 74-77 (2014).

3. Li, H. et al. Hypercytotoxicity and Rapid Loss of NKp44+ Innate Lymphoid Cells during Acute SIV Infection. PLoS Pathogens10, e1004551 (2014).

4. Xu, H., Wang, X., Lackner, A. A. \& Veazey, R. S. Type 3 innate lymphoid cell depletion is mediated by TLRs in lymphoid tissues of simian immunodeficiency virus-infected macaques. The FASEB Journal29, 5072-5080 (2015).

5. Ploquin, M. J., Silvestri, G. \& Müller-Trutwin, M. Immune activation in HIV infection: what can the natural hosts of simian immunodeficiency virus teach us? Curr Opin HIV AIDS11, 201-208 (2016). 
6. Gordon, S. N. et al. Severe depletion of mucosal CD4+ T cells in AIDS-free simian immunodeficiency virus-infected sooty mangabeys. J. Immunol.179, 3026-3034 (2007).

7. Pandrea, I. V. et al. Acute loss of intestinal CD4+ T cells is not predictive of simian immunodeficiency virus virulence. J. Immunol.179, 3035-3046 (2007).

8. Estes, J. D. et al. Damaged intestinal epithelial integrity linked to microbial translocation in pathogenic simian immunodeficiency virus infections. PLoS Pathog.6, e1001052 (2010).

9. Brenchley, J. M. et al. Differential Th17 CD4 T-cell depletion in pathogenic and nonpathogenic lentiviral infections. Blood112, 2826-2835 (2008).

10. Diop, O. M. et al. High levels of viral replication during primary simian immunodeficiency virus SIVagm infection are rapidly and strongly controlled in African green monkeys. J. Virol.74, 7538-7547 (2000).

11. Estes, J. D., Haase, A. T. \& Schacker, T. W. The role of collagen deposition in depleting CD4+ T cells and limiting reconstitution in HIV-1 and SIV infections through damage to the secondary lymphoid organ niche. Semin. Immunol.20, 181-186 (2008).

12. Li, Y. et al. SIV Infection of Lung Macrophages. PLOS ONE10, e0125500 (2015).

13. El Costa, H. et al. The local environment orchestrates mucosal decidual macrophage differentiation and substantially inhibits HIV-1 replication. Mucosal Immunol9, 634-646 (2016).

14. Arrildt, K. T. et al. Phenotypic Correlates of HIV-1 Macrophage Tropism. Journal of Virology89, 11294-11311 (2015).

15. Goldstein, S. et al. Wide range of viral load in healthy african green monkeys naturally infected with simian immunodeficiency virus. J. Virol.74, 11744-11753 (2000). 
16. Milush, J. M. et al. Lack of clinical AIDS in SIV-infected sooty mangabeys with significant CD4+ $\mathrm{T}$ cell loss is associated with double-negative $\mathrm{T}$ cells. J. Clin. Invest.121, 1102-1110 (2011).

17. Klatt, N. R. et al. Availability of activated CD4+ T cells dictates the level of viremia in naturally SIV-infected sooty mangabeys. J. Clin. Invest.118, 2039-2049 (2008).

18. Ortiz, A. M. et al. Depletion of $\mathrm{CD}^{+} \mathrm{T}$ cells abrogates post-peak decline of viremia in SIV-infected rhesus macaques. J. Clin. Invest.121, 4433-4445 (2011).

19. Micci, L. et al. CD4 depletion in SIV-infected macaques results in macrophage and microglia infection with rapid turnover of infected cells. PLoS Pathog.10, e1004467 (2014).

20. Mir, K. D. et al. Reduced Simian Immunodeficiency Virus Replication in Macrophages of Sooty Mangabeys Is Associated with Increased Expression of Host Restriction Factors. Journal of Virology89, 10136-10144 (2015).

21. Sandler, N. G. \& Douek, D. C. Microbial translocation in HIV infection: causes, consequences and treatment opportunities. Nat. Rev. Microbiol.10, 655-666 (2012).

22. Westmoreland, S. V. et al. SIV vpx is essential for macrophage infection but not for development of AIDS. PLoS ONE9, e84463 (2014).

23. Kuller, L. H. et al. Inflammatory and coagulation biomarkers and mortality in patients with HIV infection. PLoS Med.5, e203 (2008).

24. Tenorio, A. R. et al. Soluble markers of inflammation and coagulation but not T-cell activation predict non-AIDS-defining morbid events during suppressive antiretroviral treatment. J. Infect. Dis.210, 1248-1259 (2014).

25. León, A. et al. Association of microbial translocation biomarkers with clinical outcome in controllers HIV-infected patients. AIDS29, 675-681 (2015). 
26. Brenchley, J. M. Mucosal immunity in human and simian immunodeficiency lentivirus infections. Mucosal Immunol6, 657-665 (2013).

27. Baker, J. V. et al. Immunologic predictors of coronary artery calcium progression in a contemporary HIV cohort. AIDS28, 831-840 (2014).

28. Hasegawa, A. et al. The level of monocyte turnover predicts disease progression in the macaque model of AIDS. Blood114, 2917-2925 (2009).

29. Burdo, T. H. et al. Increased monocyte turnover from bone marrow correlates with severity of SIV encephalitis and CD163 levels in plasma. PLoS Pathog.6, e1000842 (2010).

30. Mir, K. D. et al. Simian immunodeficiency virus-induced alterations in monocyte production of tumor necrosis factor alpha contribute to reduced immune activation in sooty mangabeys. J. Virol.86, 7605-7615 (2012).

31. Into the wild: simian immunodeficiency virus (SIV) infection in natural hosts. - PubMed NCBI. Available at: http://www.ncbi.nlm.nih.gov/pubmed/18676179. (Accessed: 17th May 2016)

32. Ma, D. et al. SIVagm infection in wild African green monkeys from South Africa: epidemiology, natural history, and evolutionary considerations. PLoS Pathog.9, e1003011 (2013).

33. Wijewardana, V. et al. Kinetics of myeloid dendritic cell trafficking and activation: impact on progressive, nonprogressive and controlled SIV infections. PLoS Pathog.9, e1003600 (2013).

34. Jochems, S. P. et al. Modulation of Type I Interferon-Associated Viral Sensing during Acute Simian Immunodeficiency Virus Infection in African Green Monkeys. Journal of Virology89, 751-762 (2015). 
35. Bruel, T. et al. Plasmacytoid dendritic cell dynamics tune interferon-alfa production in SIV-infected cynomolgus macaques. PLoS Pathog.10, e1003915 (2014).

36. Brown, K. N., Wijewardana, V., Liu, X. \& Barratt-Boyes, S. M. Rapid influx and death of plasmacytoid dendritic cells in lymph nodes mediate depletion in acute simian immunodeficiency virus infection. PLoS Pathog.5, e1000413 (2009).

37. Donaghy, H., Gazzard, B., Gotch, F. \& Patterson, S. Dysfunction and infection of freshly isolated blood myeloid and plasmacytoid dendritic cells in patients infected with HIV-1. Blood101, 4505-4511 (2003).

38. Jochems, S. P. et al. Plasmacytoid Dendritic Cell Infection and Sensing Capacity during Pathogenic and Nonpathogenic Simian Immunodeficiency Virus Infection. J. Virol.89, 6918-6927 (2015).

39. Jacquelin, B. et al. Nonpathogenic SIV infection of African green monkeys induces a strong but rapidly controlled type I IFN response. J. Clin. Invest.119, 3544-3555 (2009).

40. Bosinger, S. E. et al. Intact type I Interferon production and IRF7 function in sooty mangabeys. PLoS Pathog.9, e1003597 (2013).

41. Diop, O. M. et al. Plasmacytoid dendritic cell dynamics and alpha interferon production during Simian immunodeficiency virus infection with a nonpathogenic outcome. $J$. Virol.82, 5145-5152 (2008).

42. Jacquelin, B. et al. Innate immune responses and rapid control of inflammation in African green monkeys treated or not with interferon-alpha during primary SIVagm infection. PLoS Pathog.10, e1004241 (2014).

43. Sandler, N. G. et al. Type I interferon responses in rhesus macaques prevent SIV infection and slow disease progression. Nature511, 601-605 (2014).

44. Bosinger, S. E. et al. Global genomic analysis reveals rapid control of a robust innate response in SIV-infected sooty mangabeys. J. Clin. Invest.119, 3556-3572 (2009). 
45. Manches, O. \& Bhardwaj, N. Resolution of immune activation defines nonpathogenic SIV infection. J. Clin. Invest.119, 3512-3515 (2009).

46. Laguette, N. et al. SAMHD1 is the dendritic- and myeloid-cell-specific HIV-1 restriction factor counteracted by Vpx. Nature474, 654-657 (2011).

47. Fonteneau, J.-F. et al. Human immunodeficiency virus type 1 activates plasmacytoid dendritic cells and concomitantly induces the bystander maturation of myeloid dendritic cells. J. Virol.78, 5223-5232 (2004).

48. Eberl, G., Colonna, M., Di Santo, J. P. \& McKenzie, A. N. J. Innate lymphoid cells: A new paradigm in immunology. Science348, aaa6566-aaa6566 (2015).

49. Yang, Q. \& Bhandoola, A. The development of adult innate lymphoid cells. Current Opinion in Immunology39, 114-120 (2016).

50. Walker, J. A., Barlow, J. L. \& McKenzie, A. N. J. Innate lymphoid cells--how did we miss them? Nat. Rev. Immunol.13, 75-87 (2013).

51. Németh, T. \& Mócsai, A. Feedback Amplification of Neutrophil Function. Trends Immunol. (2016). doi:10.1016/j.it.2016.04.002

52. Tecchio, C. \& Cassatella, M. A. Neutrophil-derived chemokines on the road to immunity. Semin. Immunol. (2016). doi:10.1016/j.smim.2016.04.003

53. Elbim, C. et al. The role of phagocytes in HIV-related oxidative stress. J. Clin. Virol.20, 99-109 (2001).

54. Casulli, S. \& Elbim, C. Interactions between human immunodeficiency virus type 1 and polymorphonuclear neutrophils. J Innate Immun6, 13-20 (2014).

55. Elbim, C., Katsikis, P. D. \& Estaquier, J. Neutrophil apoptosis during viral infections. Open Virol J3, 52-59 (2009). 
56. Elbim, C. et al. Early divergence in neutrophil apoptosis between pathogenic and nonpathogenic simian immunodeficiency virus infections of nonhuman primates. $J$. Immunol.181, 8613-8623 (2008).

57. Elbim, C. et al. Increased neutrophil apoptosis in chronically SIV-infected macaques. Retrovirology6, 29 (2009).

58. Amano, K. et al. Neutrophils induced licensing of natural killer cells. Mediators Inflamm.2015, 747680 (2015).

59. Costantini, C. \& Cassatella, M. A. The defensive alliance between neutrophils and NK cells as a novel arm of innate immunity. J. Leukoc. Biol.89, 221-233 (2011).

60. Jaeger, B. N. et al. Neutrophil depletion impairs natural killer cell maturation, function, and homeostasis. J. Exp. Med.209, 565-580 (2012).

61. Bandyopadhyay, K., Marrero, I. \& Kumar, V. NKT cell subsets as key participants in liver physiology and pathology. Cell. Mol. Immunol.13, 337-346 (2016).

62. Gapin, L. Development of invariant natural killer T cells. Curr. Opin. Immunol.39, 68-74 (2016).

63. Matsuda, J. L. et al. Natural killer T cells reactive to a single glycolipid exhibit a highly diverse $\mathrm{T}$ cell receptor beta repertoire and small clone size. Proc. Natl. Acad. Sci. U.S.A.98, 12636-12641 (2001).

64. Lee, P. T., Benlagha, K., Teyton, L. \& Bendelac, A. Distinct functional lineages of human V(alpha)24 natural killer T cells. J. Exp. Med.195, 637-641 (2002).

65. Takahashi, T. et al. Cutting edge: analysis of human V alpha $24+\mathrm{CD} 8+\mathrm{NK} \mathrm{T}$ cells activated by alpha-galactosylceramide-pulsed monocyte-derived dendritic cells. $J$. Immunol.168, 3140-3144 (2002).

66. Ishihara, S. et al.CD8(+)NKR-P1A (+)T cells preferentially accumulate in human liver. Eur. J. Immunol.29, 2406-2413 (1999). 
67. Fernandez, C. S. et al. Peripheral NKT cells in simian immunodeficiency virus-infected macaques. J. Virol.83, 1617-1624 (2009).

68. Rout, N. et al. Paucity of CD4+ natural killer T (NKT) lymphocytes in sooty mangabeys is associated with lack of NKT cell depletion after SIV infection. PLoS ONE5, e9787 (2010).

69. Rout, N. et al. Loss of effector and anti-inflammatory natural killer T lymphocyte function in pathogenic simian immunodeficiency virus infection. PLoS Pathog.8, e1002928 (2012).

70. Campillo-Gimenez, L. et al. AIDS progression is associated with the emergence of IL-17producing cells early after simian immunodeficiency virus infection. J. Immunol.184, 984-992 (2010).

71. Hirai, T. et al. Clonal Deletion Established via Invariant NKT Cell Activation and Costimulatory Blockade Requires In Vivo Expansion of Regulatory T Cells. Am. J. Transplant.16, 426-439 (2016).

72. Reeves, R. K. et al. Antigen-specific NK cell memory in rhesus macaques. Nat. Immunol.16, 927-932 (2015).

73. Schafer, J. L., Li, H., Evans, T. I., Estes, J. D. \& Reeves, R. K. Accumulation of Cytotoxic CD16+ NK Cells in Simian Immunodeficiency Virus-Infected Lymph Nodes Associated with In Situ Differentiation and Functional Anergy. J. Virol.89, 6887-6894 (2015).

74. Pereira, L. E., Johnson, R. P. \& Ansari, A. A. Sooty mangabeys and rhesus macaques exhibit significant divergent natural killer cell responses during both acute and chronic phases of SIV infection. Cell. Immunol.254, 10-19 (2008).

75. Stacey, A. R. et al. Induction of a striking systemic cytokine cascade prior to peak viremia in acute human immunodeficiency virus type 1 infection, in contrast to more modest and 
delayed responses in acute hepatitis B and C virus infections. J. Virol.83, 3719-3733 (2009).

76. Mueller, Y. M. \& Katsikis, P. D. IL-15 in HIV infection: pathogenic or therapeutic potential? Eur. Cytokine Netw.21, 219-221 (2010).

77. Baumgarth, N. The double life of a B-1 cell: self-reactivity selects for protective effector functions. Nat. Rev. Immunol.11, 34-46 (2011).

78. Mucida, D. et al. Transcriptional reprogramming of mature $\mathrm{CD} 4^{+}$helper $\mathrm{T}$ cells generates distinct MHC class II-restricted cytotoxic T lymphocytes. Nat. Immunol.14, 281-289 (2013).

79. Warren, W. C. et al. The genome of the vervet (Chlorocebus aethiops sabaeus). Genome Res.25, 1921-1933 (2015).

80. Bosinger, S. E., Jacquelin, B., Benecke, A., Silvestri, G. \& Müller-Trutwin, M. Systems biology of natural simian immunodeficiency virus infections. Curr Opin HIV AIDS7, 7178 (2012).

81. Galván-Peña, S. \& O’Neill, L. A. J. Metabolic reprograming in macrophage polarization. Front Immunol5, 420 (2014).

82. El Kasmi, K. C. \& Stenmark, K. R. Contribution of metabolic reprogramming to macrophage plasticity and function. Semin. Immunol.27, 267-275 (2015).

83. Saez-Cirion, A., Jacquelin, B., Barré-Sinoussi, F. \& Müller-Trutwin, M. Immune responses during spontaneous control of HIV and AIDS: what is the hope for a cure? Philos. Trans. R. Soc. Lond., B, Biol. Sci.369, 20130436 (2014). 


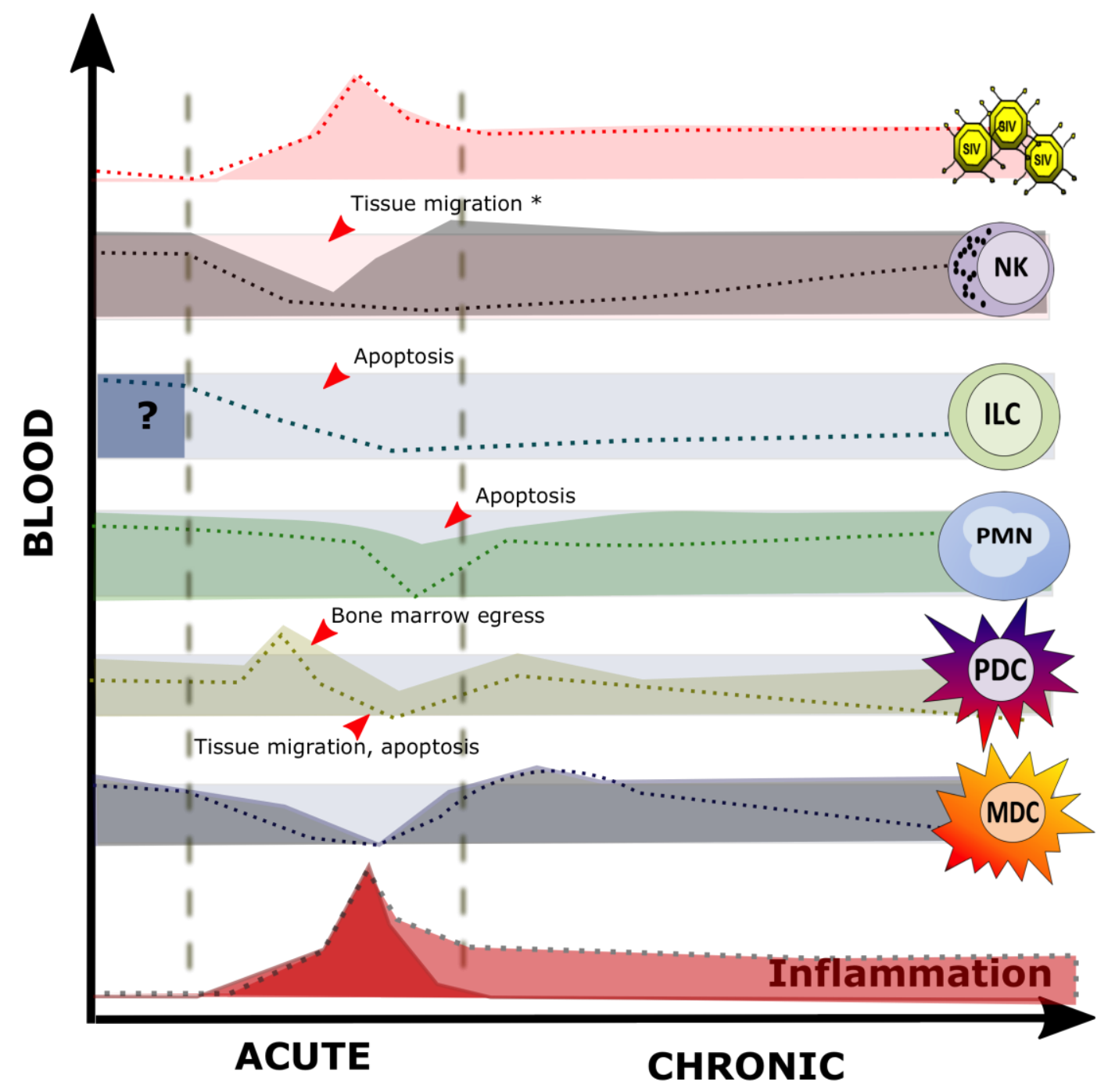

